\title{
Ablación mediante radiofrecuencia intersticial (RITA) en el tratamiento del cáncer de próstata órgano-confinado
}

\author{
Bonillo García MA, Gimeno Argente V, Jiménez Cruz JF. \\ Servicio de Urología. Hospital Universitario La Fe. Valencia.
}

Actas Urol Esp. 2007;31(6):627-632

\section{RESUMEN}

\section{ABLACIÓN MEDIANTE RADIOFRECUENCIA INTERSTICIAL (RITA) EN EL TRATAMIENTO DEL CÁNCER DE PRÓSTATA ÓRGANO-CONFINADO}

La ablación mediante radiofrecuencia (RF) permite una destrucción irreversible de tejido prostático al generar temperaturas cercanas a los $100^{\circ} \mathrm{C}$ que inducen una necrosis coagulativa de la zona tumoral y, en base a los estudios preliminares realizados en los últimos años, se ha mostrado como una alternativa terapéutica segura y eficaz en el tratamiento del cáncer de próstata órgano-confinado. Es una modalidad terapéutica minimanente invasiva, con escasas complicaciones y especialmente atractiva en aquellos pacientes en los que su co-morbilidad excluye otras opciones de tratamiento que requieran anestesia general u hospitalización. Otros aspectos interesantes de esta modalidad de tratamiento son el escaso tiempo de ablación (alrededor de 10 minutos), la posibilidad de controlar el tamaño de las lesiones inducidas, la capacidad de ser una técnica fácilmente reproducible por cualquier urólogo, ya que sólo requiere una experiencia básica en técnicas de imagen y la posibilidad de repetir el tratamiento si es preciso. A pesar de estos resultados prometedores, serían necesarios estudios a largo plazo antes de pronunciarnos de forma definitiva sobre la eficacia del RITA y determinar su lugar exacto en el tratamiento del cáncer de próstata órgano-confinado.

Palabras claves: Cáncer de próstata. Procedimiento minimamente invasivo. Radioterapia.

\section{ABSTRACT \\ RADIOFREQUENCY INTERSTITIAL TUMOR ABLATION (RITA) FOR THE TREATMENT OF LOCALISED PROSTATE CANCER}

Radiofrequency ablation produces an irreversible destruction of prostate tissue since it reaches temperatures of $110^{\circ} \mathrm{C}$ that leads the tumour into coagulative necrosis and, based on preliminary studies, it has shown to be a safe therapeutical alternative for localised prostate cancer. It is a non-invasive technique with scarce complications which is especially attractive for those patients whose co-morbidity precludes them from having any others procedures that require general anaesthesia or that make them to be admitted. Another valuable aspect of this treatment modality is that treatment times are short (less than 10 minutes), and controlled lesions can be predictably generated, as well as it is a feasible technique by most urologists since it requires only basic imaging experience and it could be also repeated at any time if needed. However, despite these promising results, long term follow-up are needed before conclusions about the efficacy of RITA can be drawn and, therefore, before setting its role in localised prostate cancer treatment.

Keywords: Prostate cancer. Minimally invasive procedure. Radiotherapy.

$\mathrm{E}^{\prime}$ cáncer de próstata constituye la neoplasia más frecuente y la segunda causa de muerte por tumor en el hombre. Debido a la inclusión de la determinación del antígeno prostático específico (PSA) sérico en la práctica clínica, la epide- miologia de esta neoplasia ha cambiado dramáticamente, estimando algunos autores que el $4 \%$ de los casos nuevos de cáncer de próstata diagnosticados corresponden a varones menores de 50 años, a diferencia del $1 \%$ en la era pre-PSA ${ }^{1}$. 
Aún más, de los nuevos casos diagnosticados, se cree que, alrededor del $80 \%$, se encuentra limitado a la glándula prostática (órgano-confinado) ${ }^{2}$.

Clásicamente, se ha considerado a la prostatectomía radical (PR) y a la radioterapia externa (RT), como tratamiento de elección del cáncer de próstata órgano-confinado. Sin embargo, las complicaciones asociadas y el hallazgo, no poco frecuente, de enfermedad extracapsular en la pieza quirúrgica, han impulsado que, en las últimas décadas, se hayan desarrollado alternativas terapéuticas que pretenden minimizar la morbilidad inherente a estos abordajes terapéuticos. En este sentido, se han popularizado en la literatura médica resultados de nuevas opciones como la crioterapia, la braquiterapia, la radiofrecuencia y el uso de ultrasonidos (HIFU).

La ablación mediante radiofrecuencia (RF) ha demostrado ser un método eficaz y seguro en el tratamiento de hepatocarcinoma ${ }^{3}$, metástasis hepáticas y osteomas osteoides ${ }^{4}$. También, se ha propuesto como alternativa terapéutica frente a la cirugía conservadora renal en el tratamiento del cáncer de riñón ${ }^{5}$. La RF intersticial permite una destrucción irreversible de tejido vivo al generar temperaturas cercanas a los $100{ }^{\circ} \mathrm{C}$ que inducen una necrosis coagulativa de la zona tumoral, sin evidencia de trombosis venosa o hemorragias significativas en los bordes tumorales ${ }^{6}$. Este tipo de energia ha sido empleada extensamente en el tratamiento de la hiperplasia benigna de próstata (HBP) mediante un sistema de ablación transuretral (TUNA), al provocar una destrucción tisular localizada alrededor de las agujas insertadas en la próstata7 ${ }^{7}$. Sin embargo, si tenemos en cuenta que el cáncer de próstata habitualmente se localiza en la zona periférica y que se trata de una enfermedad multifocal, el tratamiento en este caso no podrá limitarse a la destrucción de la lesión visible o palpable, sino que requerirá la destrucción de toda la glándula.

\section{PROCEDIMIENTO}

Según la técnica inicial descrita por Zlotta ${ }^{8}$, se coloca al paciente en posición de litotomía bajo anestesia general o raquídea. Otras publicaciones describen haber realizado el procedimiento con anestesia local $^{6}$. Se administra profilaxis antimicrobiana y preparación intestinal durante el preoperatorio. Antes de comenzar el tratamiento, se procede a la colocación de una sonda uretral de Foley con triple vía para irrigar suero salino frío (15\%) a un ritmo de $50 \mathrm{cc} / \mathrm{min}$ para enfriar la uretra, y a continuación mediante una sonda ecográfica transrectal se miden los diámetros transverso, antero-posterior y craneocaudal de la glándula prostática. De igual modo, al paciente se coloca alrededor de la cadera una especie de toma de tierra.

La energía es producida por un generador de RF (Fig. 1) que puede alcanzar los $50 \mathrm{~W}$ a una frecuencia de $460 \mathrm{kHz}$. Se administra mediante una aguja de 15-gauge que presenta en su extremo distal 3 electrodos monopolares en forma de garfio, que se encuentran separados entre ellos con un ángulo de $120^{\circ} \mathrm{y}$ al desplegarse describen una esfera de $2 \mathrm{~cm}$ de volumen. A su vez, en los extremos de cada uno de los garfios se localiza un dispositivo para registrar la temperatura alcanzada durante el procedimiento. Cada aguja cuenta con un mecanismo que regula la impedancia de la lesión si se precisara, y la vaina de dicha aguja se encuentra aislada a excepción del centímetro distal que actúa también como un electrodo activo. La impedancia, que es un factor fundamental en el tamaño y evolución de la lesión térmica, expresa el grado de hidratación tisular y su capacidad para transmitir el calor. Al ser también reflejo del área sobre la que actúa el electrodo, una superficie grande tendrá una impedancia baja, mientras que superficies pequeñas tendrán impedancias altas.

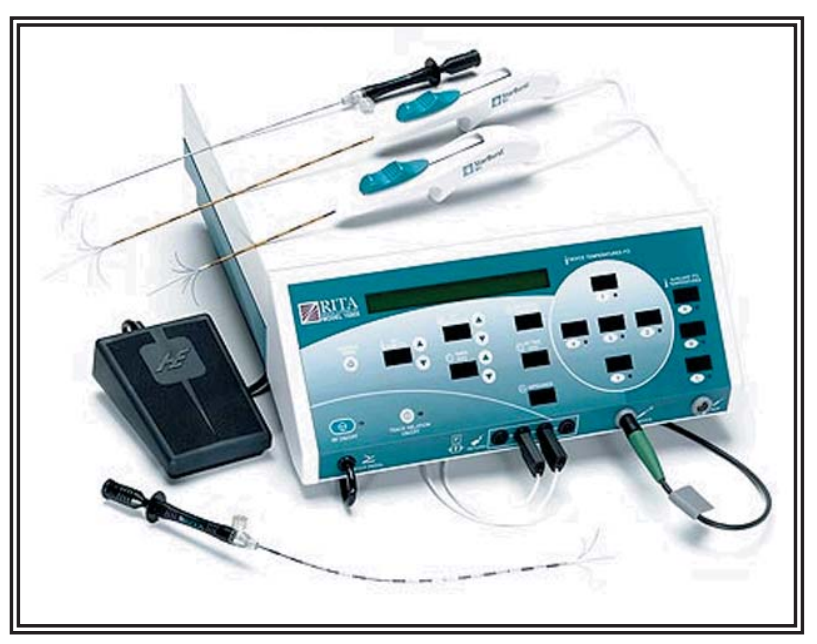

FIGURA 1. Generador de RF con agujas con electrodos monopolares. 
Se realiza una pequeña incisión en el periné, y a continuación se introduce transperinealmente la aguja bajo control ecográfico, hasta alcanzar la localización deseada en uno de los lóbulos prostáticos. Mediante la ecografía prostática transrectal la aguja es posicionada de forma precisa en tiempo real, tanto en su plano transversal como sagital. El generador ofrece en cada momento información a cerca de la impedancia del sistema, la cantidad total de energía suministrada y de la temperatura de cada garfio por separado. Además, se recoge la lectura térmica de 3 dispositivos situados en una misma aguja con una separación de $5 \mathrm{~mm}$ colocada en el plano entre la próstata y el recto. Se comienza con una energía de $10 \mathrm{~W}$ y se diseña para que se alcance $100^{\circ} \mathrm{C}$ en cada uno de los garfios en 2-3 minutos y posteriormente, se mantiene esta temperatura durante otros 3 minutos. Esta cantidad de energía puede ser regulada en todo momento por quién está realizando el procedimiento en función del objetivo establecido.

La ablación con RF con la técnica RITA destruye la glándula prostática mediante el calor que produce el electrodo y su posterior conducción a los tejidos adyacentes. Utiliza una corriente alterna de alta frecuencia que produce una agitación iónica o molecular generada por la energía térmica, distribuyéndose alrededor del electrodo para formar una lesión esférica. El efecto es directamente proporcional a la intensidad de la corriente alterna y, asumiendo homogeneidad física y eléctrica, el calor generado y por lo tanto el tamaño de la lesión, varían directamente con el tiempo de la aplicación de la corriente de RF e inversamente al flujo sanguíneo ${ }^{9}$. Si la energía es muy alta, provocaremos una rápida deshidratación de los tejidos con el correspondiente incremento de la impedancia e impediremos el efecto de la RF. Por lo tanto, el tamaño de la lesión creada por RF se puede predecir según las características físicas de la energía aplicada, obligándonos a encontrar un equilibrio entre la intensidad para crear una lesión controlada y aquella que provocaría un incremento de la impedancia que chamuscaría los tejidos de alrededor de la aguja.

Tras este proceso es fundamental un periodo de enfriamiento (cooldown cycle) que verifique si el procedimiento ha sido eficaz. Durante este tiempo, se apaga el generador y se registra de forma separada la temperatura de cada garfio durante 60 segundos. Basándose en estudios previos con RF sobre tejido humano, Djavan et al. ${ }^{6}$ consideran que una caída de la temperatura por debajo de los 55ㅇ $\mathrm{C}$ en 60 segundos predice una lesión incompleta. La explicación está basada en que el calor se disipa, independientemente de la fuente, por conducción y convección. La conducción es el calentamiento y enfriamiento de las células y tejidos adyacentes que tiene lugar al alejarte de la fuente de calor. Por lo tanto, existe un obvio descenso de la temperatura del tejido conforme aumentamos la distancia $\left(1 / \mathrm{r}^{4}\right)$. En un modelo experimental perfecto se produce una pérdida suave y gradual del gradiente de temperatura que indicaría un "sellado" de la lesión. Si, en algún punto previo a la llegada de los límites tumorales ocurre una caída brusca de la temperatura, no representaría una pérdida por conducción sino más bien por convección, al persistir tejido vascularizado. Esta caída brusca representa la persistencia de tejido viable y vascularizado. Por tanto, basados en este fenómeno, más que las imágenes ecográficas, el periodo de enfriamiento es fundamental a la hora de verificar intraoperatoriamente si el procedimiento realizado ha sido o no completo.

\section{COMPLICACIONES}

Dado que la longitud de onda de la RF es muy larga para crear lesiones no deseadas a distancia, no se contemplan en la literatura revisa$\mathrm{da}^{1,6,8,10,11}$ efectos secundarios importantes sobre órganos vecinos; consecuentemente, el electrodo situado en el plano entre el recto y la próstata no registra un incremento importante de la temperatura durante el procedimiento ${ }^{12}$.

\section{SEGUIMIENTO}

Los pacientes tratados con RITA deben ser evaluados mediante tacto rectal y determinación sérica de PSA cada 3 meses durante el primer año y bianualmente con posterioridad.

Adicionalmente al seguimiento clínico estándar, la eficacia de este abordaje terapéutico puede analizarse mediante biopsia de próstata transrectal a los 6 y 12 meses postratamiento, 
mostrando la existencia de necrosis coagulativa y atrofia en los tejidos sometidos a tratamiento (Fig. 2). De igual forma, podemos utilizar técnicas de imagen como la ecografía power-doppler (Fig. 3), que revelará la ausencia de flujo sanguíneo en las zonas de próstata tratadas con RITA o la resonancia magnética (Fig. 4), donde podremos observar cambios en la intensidad de la señal de aquellas áreas sometidas a este tratamiento.

\section{DISCUSIÓN}

La necesidad de alternativas terapéuticas mínimamente invasivas en el tratamiento del cáncer de próstata localizado, surge cuando analizamos en profundidad los tratamientos clásicos de esta patología, es decir, la prostatectomía radical y la radioterapia externa.

La prostatectomía radical es una excelente opción terapéutica, sin embargo no está exenta de inconvenientes como puedan ser: una estancia hospitalaria prolongada al tratarse de una técnica invasiva, una tasa de incontinencia tras prostatectomía entre 5-10\%, según autores, así como una tasa de impotencia por encima del $50 \%$. A esto habría que añadirle el hecho que un porcentaje no desdeñable de pacientes con cáncer de próstata localizado, presentan enfermedad localmente avanzada con hallazgo de afectación extracapsular en la pieza quirúrgica.

La radioterapia externa es un procedimiento no invasivo, pero con resultados oncológicos inferiores a los obtenidos por la prostatectomía radical. La técnica de radioterapia conformacional, que permite la administración de dosis por encima de los $80 \mathrm{~Gy}$, parece ser muy prometedora, sin embargo no disponemos de datos a largo plazo que la avalen.

Las alternativas terapéuticas mencionadas con anterioridad y entre las que se encuentra la radiofrecuencia, tienen en común que son opciones minimamente invasivas por lo que requieren estancias hospitalarias muy cortas. En general, son procedimientos seguros, bien tolerados por el paciente y con escasas complicaciones. En lo que se refiere a la eficacia terapéutica sólo está ampliamente demostrada en la braquiterapia, mientras que para el HIFU y la RF no disponemos de estudios a largo plazo por tratarse de modalidades recientemente introducidas en el tratamiento del cáncer de próstata.

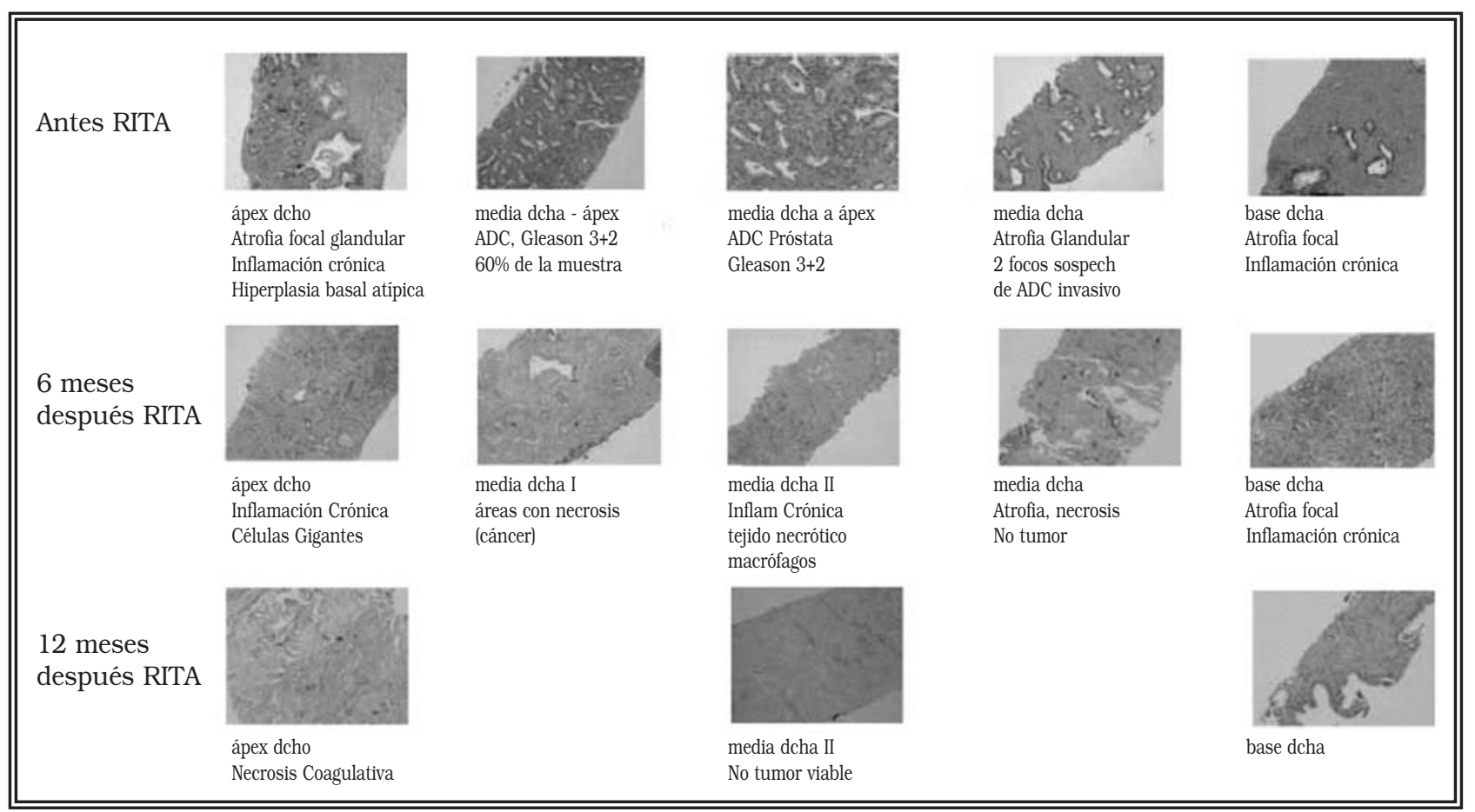

FIGURA 2. Biopsia sextante de próstata, antes y a los 6 y 12 meses después de ablación con RITA. Notar la atrofia y la necrosis coagulativa de los tejidos tratados. 


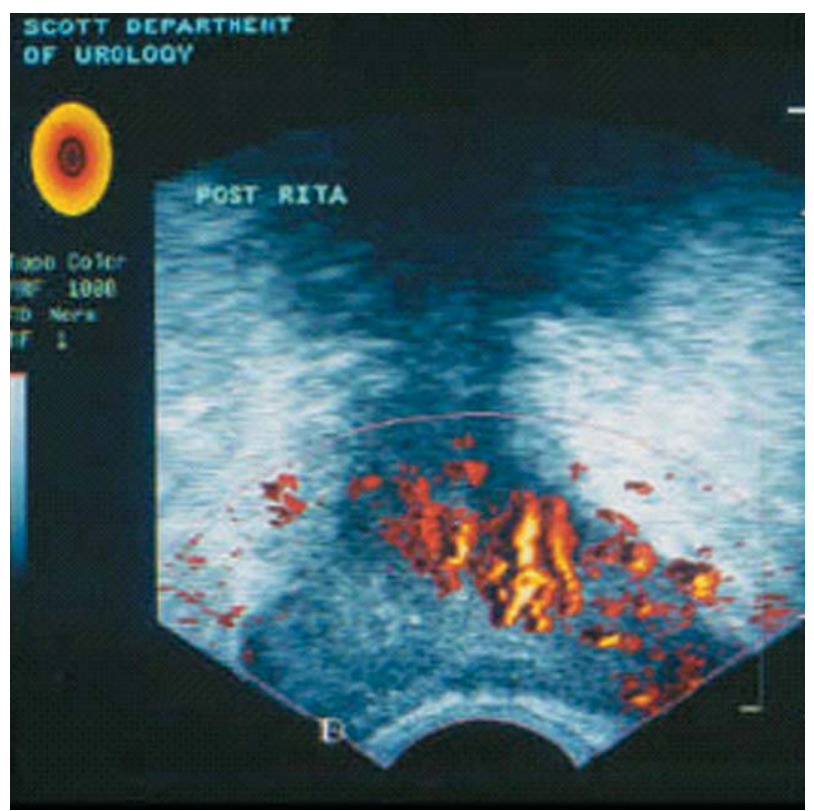

FIGURA 3. Ejemplo de ecografia power-doppler prostática inmediatamente tras el tratamiento con RITA. Notar la ausencia de flujo sanguíneo en las áreas sometidas a tratamiento con RITA.

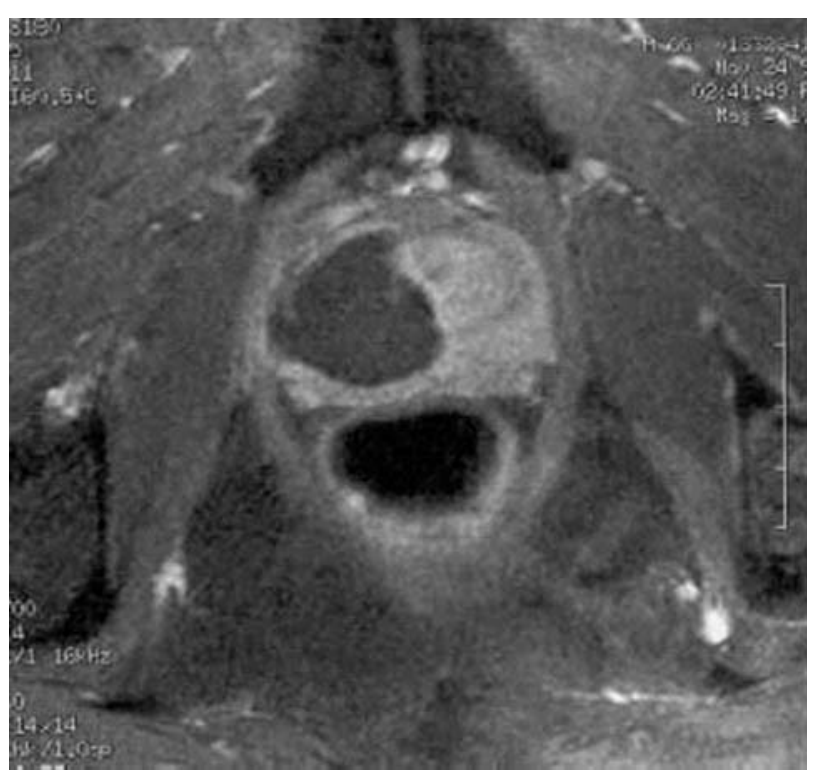

FIGURA 4. Ejemplo de resonancia magnética prostática 1 mes después del tratamiento con RITA. Notar los cambios de intensidad de señal en las zonas tratadas con RITA.

Zlotta et $\mathrm{al}^{8}$. fue quién por primera vez evaluó la eficacia y seguridad de la RF en el cáncer de próstata órgano-confinado, aplicando RITA en 15 pacientes programados para una prostatectomía radical. Concluye que el procedimiento fue bien tolerado por los pacientes y que no se registró ninguna complicación relevante. Cuando analizaron la pieza quirúrgica, las lesiones necróticas bien delimitadas se correlacionaban con el tamaño estimado del tumor. Este hallazgo es reforzado por Djavan et $\mathrm{al}^{6}$, los cuales estudiaron la correlación entre la imagen de la resonancia magnética (RM) endorrectal y el examen histopatológico en 10 pacientes motivados por la inexactitud de los hallazgos ecográficos a la hora de distinguir tejido viable del necrótico. Concluyen asegurando que RITA es un procedimiento eficaz y seguro, y que las lesiones son predecibles en tamaño y localización, al igual que la imagen obtenida mediante RM tiene buena correlación con el área necrótica de la pieza histológica, sin encontrar diferencias estadísticamente significativas entre los hallazgos radiológicos frente a los anátomo-patológicos.

Shariat et al. ${ }^{11}$ en un estudio recientemente publicado, analizan la fiabilidad, seguridad, morbilidad y eficacia preliminar del tratamiento con RITA, en 11 pacientes con recurrencia local de cáncer de próstata en los que fracasó la radioterapia local o no fueron candidatos para ningún tratamiento curativo. Confirman que RITA es una terapia fiable, segura y con escasas complicaciones (todas ellas menores). Además aseguran que este tratamiento modifica la historia natural de progresión de PSA, como demuestra el descenso de sus niveles séricos en todos los pacientes tratados, y no provoca sintomatología sobre el tracto urinario inferior.

\section{CONCLUSIONES}

La ablación mediante radiofrecuencia con la técnica RITA, en base a los estudios preliminares realizados en los últimos años, se ha mostrado como una alternativa terapéutica segura y eficaz en el tratamiento del cáncer de próstata órganoconfinado. Es una modalidad terapéutica mínimamente invasiva, con escasas complicaciones y especialmente atractiva en aquellos pacientes en los que su co-morbilidad excluye otras opciones de tratamiento que requieran anestesia general $\mathrm{u}$ hospitalización. Otros aspectos interesantes de esta modalidad de tratamiento son el escaso tiempo de ablación (alrededor de 10 minutos), la posibilidad de controlar el tamaño de las lesiones inducidas $^{6,8}$, la capacidad de ser una técnica fácil- 
mente reproducible por cualquier urólogo ya que sólo requiere una experiencia básica en técnicas de imagen y la posibilidad de repetir el tratamiento si es preciso. A pesar de estos resultados prometedores, serían necesarios estudios a largo plazo antes de pronunciarnos de forma definitiva sobre la eficacia del RITA y determinar su lugar exacto en el tratamiento del cáncer de próstata órgano-confinado.

\section{REFERENCIAS}

1. Stephenson RA. Prostate cancer trends in the era of prostate-specific antigen. An update of incidence, mortality, and clinical factors from the SEER database. Urol Clin North Am. 2002;29(1):173-181.

2. Jemal A, Murray T, Samuels A, Ghafoor A, Ward E, Thun MJ. Cancer statistics, 2003. CA Cancer J Clin. 2003;53(1): 5-26.

3. Rossi S, Di Stasi M, Buscarini E, Cavanna L, Quaretti P, Squassante E, et al. Percutaneous Radiofrequency Interstitial Thermal Ablation in the Treatment of Small Hepatocellular Carcinoma. Cancer J Sci Am. 1995;1(1):7381.

4. de Berg JC, Pattynama PM, Obermann WR, Bode PJ, Vielvoye GJ, Taminiau AH. Percutaneous computed-tomography-guided thermocoagulation for osteoid osteomas. Lancet. 1995;346(8971):350-351.

5. Corwin TS, Lindberg G, Traxer O, Gettman MT, Smith TG, Pearle MS, et al. Laparoscopic radiofrequency thermal ablation of renal tissue with and without hilar occlusion. J Urol. 2001;166(1):281-284.
6. Djavan B, Zlotta AR, Susani M, Heinz G, Shariat S, Silverman DE, et al. Transperineal radiofrequency interstitial tumor ablation of the prostate: correlation of magnetic resonance imaging with histopathologic examination. Urology. 1997;50(6):986-992; discussion 992-993.

7. Roehrborn CG, Issa MM, Bruskewitz RC, Naslund MJ, Oesterling JE, Perez-Marrero R, et al. Transurethral needle ablation for benign prostatic hyperplasia: 12-month results of a prospective, multicenter U.S. study. Urology. 1998;51 (3):415-421.

8. Zlotta AR, Djavan B, Matos C, Noel JC, Peny MO, Silverman $\mathrm{DE}$, et al. Percutaneous transperineal radiofrequency ablation of prostate tumour: safety, feasibility and pathological effects on human prostate cancer. Br J Urol. 1998;81(2): 265-275.

9. Organ LW. Electrophysiologic principles of radiofrequency lesion making. Appl Neurophysiol. 1976-1977;39(2):69-76.

10. Jindal G, Friedman M, Locklin J, Wood BJ. Palliative Radiofrequency Ablation for Recurrent Prostate Cancer. Cardiovasc Intervent Radiol. 2006;29(3):482-485.

11. Shariat SF, Raptidis G, Masatoschi M, Bergamaschi F, Slawin KM. Pilot study of radiofrequency interstitial tumor ablation (RITA) for the treatment of radio-recurrent prostate cancer. Prostate. 2005;65(3):260-267.

12. Djavan B, Susani M, Shariat S, Zlotta AR, Silverman DE, Schulman CC, et al. Transperineal radiofrequency interstitial tumor ablation (RITA) of the prostate. Tech Urol. 1998;4(2):103-109.

Correspondencia autor: Dr. M.A. Bonillo García Servicio de Urologia. Hospital Universitario La Fe Avda Campanar, 21 - 46009 Valencia. Tel.: 963862700 E-mail autor: miggs89@yahoo.es

Información artículo: Original 